International Journal of Pure and Applied Mathematics

Volume 115 No. 3 2017, 455-466

ISSN: 1311-8080 (printed version); ISSN: 1314-3395 (on-line version)

url: http://www.ijpam.eu

doi: 10.12732 /ijpam.v115i3.1

\title{
COMPLEX INTUITIONISTIC FUZZY NORMAL SUBGROUP
}

\author{
Rima Al-Husban ${ }^{1 \S}$, Abdul Razak Salleh ${ }^{2}$, Abd Ghafur Bin Ahmad ${ }^{3}$ \\ ${ }^{1,2,3}$ School of Mathematical Sciences \\ Faculty of Science and Technology \\ Universiti Kebangsaan Malaysia \\ 43600 UKM Bangi, Selangor DE, MALAYSIA
}

\begin{abstract}
In this paper we continue the study of complex intuitionistic fuzzy groups by introducing the notion of complex intuitionistic fuzzy normal subgroup based on complex intuitionistic fuzzy space as a generalisation of intuitionistic fuzzy normal subgroup.
\end{abstract}

AMS Subject Classification: 20N25

Key Words: complex intuitionistic fuzzy space, complex intuitionistic fuzzy subspace, complex intuitionistic fuzzy group, complex intuitionistic fuzzy subgroup

\section{Introduction}

The study of fuzzy groups was first started with the introduction of the concept of fuzzy subgroups by Rosenfeld (1971). In 1979 Anthony and Sherwood redefined fuzzy subgroups using the concept of triangular norm. In his remarkable paper Dib (1994) introduced a new approach to define fuzzy groups using his definition of fuzzy space which serves as the universal set in classical group theory. Dib remarked the absence of the fuzzy universal set and discussed some

\footnotetext{
Received: $\quad$ September 8, 2016

Revised: $\quad$ July 17,2017

Published: July 27, 2017

$\S_{\text {Correspondence author }}$
}

(c) 2017 Academic Publications, Ltd. url: www.acadpubl.eu 
problems in Rosenfeld's approach. The notion of fuzzy normal subgroup was first initiated by Abdul Razak (1996) to continue the theory of fuzzy groups obtained by Dib. Zhan and Tan (2004) introduced the concept of intuitionistic fuzzy subgroup as ageneralization of Rosenfeld's approach by adding constrains over the nonmembership functions. In 1998 Dib and Hassan introduced and discussed the fuzzy normal subgroup in a similar manner to Razak. In 2010 Fathi and Salleh introduced and discussed the intuitionistic fuzzy normal subgroup based on intuitionistic fuzzy space, Husban and Salleh (2015) introduced complex fuzzy normal subgroup based on complex fuzzy space.

In1989, Buckley incorporated the concepts of fuzzy numbers and complex numbers under the name fuzzy complex numbers (Buckley1989). This concept has become a famous research topic and a goal for many researchers (Buckley 1989; Ramot et al. 2002, 2003). However, the concept given by Buckley has different range compared to the range of Ramot et al.'s definition for complex fuzzy set Buckley's range goes to the interval [0,1], while Ramot et al.'s range extends to the unit circle in the complex plane. The concept of complex intuitionistic fuzzy set is introduced by Alkouri and Salleh in 2011 the intuitionistic fuzzy set is an extension of complex fuzzy set by adding complex-valued the comple grade to the definition of complex fuzzy set.

In this paper, we continue the study of complex intuitionistic fuzzy groups by generalising the notion of the intuitionistic fuzzy normal subgroup to the complex fuzzy normal subgroup.

\section{Preliminaries}

In this section we recall some definition which will be used in the paper .

Definition 2.1. (see Zadeh 1965) A fuzzy set $A$ in a universe of discourse $U$ is characterised by a membership function $\mu_{A}(x)$ that takes values in the interval $[0,1]$.

Definition 2.2. (see Atanassov 1986) An intuitionistic fuzzy set $A$ in a non-empty set $U$ (a universe of discourse) is an object having the form:

$$
A=\left\{\left\langle x, \mu_{A}(x), \gamma_{A}(x)\right\rangle: x \in U\right\}
$$

where the functions $\mu_{A}(x): U \rightarrow[0,1]$ and $\gamma_{A}(x): U \rightarrow[0,1]$, denote the degree of membership and degree of non-membership of each element $x \in U$ to the set $A$ respectively, and $0 \leq \mu_{A}(x)+\gamma_{A}(x) \leq 1$ for all $x \in U$. 
Definition 2.3. (see Ramot et al. 2002) A complex fuzzy set A, defined on a universe of discourse $U$, is characterised by a membership function $\mu_{A}(x)$, that assigns to any element $x \in U$ a complex-valued grade of membership in $A$. By definition, the values of $\mu_{A}(x)$, may receive all lying within the unit circle in the complex plane, and are thus of the form $\mu_{A}(x)=r_{A}(x) e^{i \omega_{A}(x)}$, where $i=\sqrt{-1}$, each of $r_{A}(x)$ and $\omega_{A}(x)$ are both real-valued, and $r_{A}(x) \in[0,1]$. The CFS $A$ may be represented as the set of ordered pairs

$$
A=\left\{\left(x, \mu_{A}(x)\right): x \in U\right\}=\left\{\left(x, r_{A}(x) e^{i \omega_{A}(x)}\right): x \in U\right\} .
$$

Definition 2.4. (see Alkouri, Salleh 2016) A complex Atanassov's intuitionistic fuzzy set $A$, defined on a universe of discourse $U$, is characterised by membership and non-membership functions $\mu_{A}(x)$ and $\gamma_{A}(x)$, respectively, that assign to any element $x \in U$ a complex-valued grade of both membership and non-membership in $A$. By definition, the values of $\mu_{A}(x), \gamma_{A}(x)$, and their sum may receive all that lies within the unit circle in the complex plane, and are of the form $\mu_{A}(x)=r_{A}(x) \cdot e^{i}, \alpha \omega_{\mu A}(x)$ for membership function $\operatorname{in} A$ and $\gamma_{A}(x)=k_{A}(x) \cdot e^{i \alpha \omega_{\gamma_{A}}(x)}$ for non-membership function in $A$, where $i=\sqrt{-1}$, each of $r_{A}(x)$ and $k_{A}(x)$ are real-valued and both belong to the interval $[0,1]$ such that $0 \leq r_{A}(x)+k_{A}(x) \leq 1$, also $\omega_{\mu_{A}}(x)$ and $\omega_{\gamma_{A}}(x)$ are real valued. We represent the A complex Atanassov's intuitionistic fuzzy set Aas

$$
A=\left\{\left\langle x, \mu_{A}(x), \gamma_{A}(x)\right\rangle: x \in U\right\}
$$

where

$$
\begin{gathered}
\mu_{A}(x): U \rightarrow\{a|a \in C,| a \mid \leq 1\}, \\
\gamma_{A}(x): U \rightarrow\left\{a^{\prime}\left|a^{\prime} \in C,\right| a^{\prime} \mid \leq 1\right\}, \quad\left|\mu_{A}(x)+\gamma_{A}(x)\right| \leq 1 .
\end{gathered}
$$

Definition 2.5. (see Al-Husban, Salleh 2016) Let $X$ be a nonempty set. A complex fuzzy space, denoted by $\left(X, E^{2}\right)$, where $E^{2}$ is the unit disc, is the set of all ordered pairs where $\left(X, E^{2}\right)=\left\{\left(x, r e^{i \theta_{r}}\right): r e^{i \theta_{r}} \in E^{2}, x \in X\right\}$, where, $i \in \sqrt{-1}, \quad r \in[0,1]$ and $\theta_{r} \in[0,2 \pi]$. The ordered pair $\left(x, E^{2}\right)$ is called a complex fuzzy element in the complex fuzzy space $\left(X, E^{2}\right)$.

Definition 2.6. (see Husban $\mathrm{R}$ and Salleh 2016) Let $X$ be a nonempty set. A complex intuitionistic fuzzy space denoted by $\left(X, E^{2}, E^{2}\right)$, where $E^{2}$ is unit disc in complex plane is the set of all ordered triples $\left(x, E^{2}, E^{2}\right)$ where $\left.\left(x, E^{2}, E^{2}\right) \in E^{2}\right\}$ with $r+s \leq 1, \alpha \in[0,1] \quad \theta_{r}, \theta_{s} \in[0,2 \pi]$ and $x \in X$. The ordered triple $\left(x, E^{2}, E^{2}\right)$ is called a complex intuitionistic fuzzy element of the complex intuitionistic fuzzy space $\left(X, E^{2}, E^{2}\right)$ and the condition, $r e^{i \theta_{r}}, s e^{i \theta_{s}} \in$ 
$E^{2}$ with $\left|r e^{i \theta_{r}}+s e^{i \theta_{s}}\right| \leq 1$ will be referred to as the complex intuitionistic condition.

Definition 2.7. (see Husban $\mathrm{R}$ and Salleh 2016) Let $U_{0}$ be the support of a given subset $U$ of $X$. A complex intuitionistic fuzzy subspace $U$ of the complex intuitionistic fuzzy space $\left(X, E^{2}, E^{2}\right)$ is the collection of all ordered triples $\left(x, \underline{r} e^{i \underline{\theta}_{r}}, \bar{r} e^{i \bar{\theta}_{r}}\right)$, where, $x \in U$ and $\underline{r} e^{i \underline{\theta}_{r}}, \bar{r} e^{i \bar{\theta}_{r}}$ are subsets of $E^{2}$ such that $r e^{i \theta_{r}}$ contains at least one element beside the zero element and $r e^{i \theta_{r}}$ contains at least one element beside the unit. If $x \notin U_{0}$, then $\underline{r}=0$ and $\underline{\theta}_{r}=0$ and $\bar{r}=1, \bar{\theta}_{r}=2 \pi$. The ordered triple $\left(x, \underline{r} e^{i \theta_{r}}, \bar{r} e^{i \bar{\theta}_{r}}\right)$ will be called a complex intuitionistic fuzzy element of the complex intuitionistic fuzzy subspace $U$. The empty complex intuitionistic fuzzy subspace denoted by $\phi$ is defined to be: $\phi=$ $\left\{\left(x, E^{2}, E^{2}\right): x \in \phi\right\}$.

Definition 2.8. (see R. Husban and Salleh 2016) A complex intuitionistic fuzzy relation $\rho$ from a complex intuitionistic fuzzy space $\left(X, E^{2}, E^{2}\right)$ to a complex intuitionistic fuzzy space $\left(Y, E^{2}, E^{2}\right)$ is a subset of the complex intuitionistic fuzzy Cartesian product $\left(X, E^{2}, E^{2}\right)\left(Y, E^{2}, E^{2}\right)$ A complex intuitionistic fuzzy relation from a complex intuitionistic fuzzy space $\left(X, E^{2}, E^{2}\right)$ into itself is called a complex intuitionistic fuzzy relation in the complex intuitionistic fuzzy space $\left(X, E^{2}, E^{2}\right)$.

Definition 2.9. (see R. Husban and Salleh 2016) A complex intuitionistic fuzzy function between two complex intuitionistic fuzzy spaces $X$ and $Y$ is a complex intuitionistic fuzzy relation $\underline{\underline{F}}$ from the $X$ to $Y$ satisfying the following conditions:

1. For every $x \in X$ with $r e^{i \theta_{r}}, s e^{i \theta_{s}} \in E^{2}$, there exists a unique element $y \in Y$ with $w e^{i \theta_{w}}, z e^{i \theta_{z}} \in E^{2}$ such that

$$
\left((x, y),\left(r e^{i \theta_{r}}, w e^{i \theta_{w}}\right),\left(s e^{i \theta_{s}}, z e^{i \theta_{z}}\right)\right) \in A,
$$

for some $A \in \underline{\underline{F}}$.

2. If

$$
\left((x, y),\left(r_{1} e^{i \theta_{r_{1}}}, w_{1} e^{i \theta_{w_{1}}}\right),\left(s_{1} e^{i \theta_{s_{1}}}, z_{1} e^{i \theta_{z_{1}}}\right)\right) \in A \in \underline{\underline{F}},
$$

and

$$
\left(\left(x, y^{\prime}\right),\left(r_{2} e^{i \theta_{r_{2}}}, w_{2} e^{i \theta_{w_{2}}}\right) \quad\left(s_{2} e^{i \theta_{s_{2}}}, z_{2} e^{i \theta_{z_{2}}}\right)\right) \in B \in \underline{\underline{F}},
$$

then $y=y^{\prime}$.

3. If

$$
\left((x, y),\left(r_{1} e^{i \theta_{r_{1}}}, w_{1} e^{i \theta_{w_{1}}}\right),\left(s_{1} e^{i \theta_{s_{1}}}, z_{1} e^{i \theta_{z_{1}}}\right)\right) \in A \in \underline{\underline{F}},
$$


and

$$
\left(\left(x, y^{\prime}\right),\left(r_{2} e^{i \theta_{r_{2}}}, w_{2} e^{i \theta_{w_{2}}}\right),\left(s_{2} e^{i \theta_{s_{2}}}, z_{2} e^{i \theta_{z_{2}}}\right)\right) \in B \in \underline{\underline{F}},
$$

then $\left(r_{1}>r_{2}\right),\left(\theta_{r_{1}}>\theta_{r_{2}}\right)$ implies $\left(w_{1}>w_{2}\right),\left(\theta_{w_{1}}>\theta_{w_{2}}\right)$ and $\left(s_{1}>s_{2}\right)$, $\left(\theta_{s_{1}}>\theta_{s_{2}}\right)$ implies $\left(z_{1}>z_{2}\right),\left(\theta_{z_{1}}>\theta_{z_{2}}\right)$.

4. If

$$
\left((x, y),\left(r_{1} e^{i \theta_{r_{1}}}, w_{1} e^{i \theta_{w_{1}}}\right),\left(s_{1} e^{i \theta_{s_{1}}}, z_{1} e^{i \theta_{z_{1}}}\right)\right) \in A \in \underline{\underline{F}}
$$

then $r=0, \theta_{r}=0$ implies $w=0, \theta_{w}=0, s=1, \theta_{s}=1$ implies $z=$ $0, \theta_{z}=0$ and $r=1, \theta_{r}=1$ implies $w=1, \theta_{w}=1, s=0, \theta_{s}=0$ implies $z=1, \theta_{z}=1$.

Conditions (i) and (ii) imply that there exists a unique (ordinary) function from $X$ to $Y$, namely $F: X \rightarrow Y$ and that for every $x \in X$ there exists unique (ordinary) functions from $E^{2}$ to $E^{2}$, namely $\underline{f}_{x}, \bar{f}_{x}: E^{2} \rightarrow E^{2}$. On the other hand conditions (iii) and (iv) are respectively equivalent to the following conditions:

1. $f_{x}, \bar{f}_{x}$ are nondecreasing on $E^{2}$.

2. $f_{x}\left(0 e^{0 i}\right)=0=\bar{f}_{x}\left(1 e^{\frac{3 \pi}{4} i}\right)$ and $f_{x}\left(1 e^{0 i}\right)=1=\bar{f}_{x}\left(1 e^{0 i}\right)$.

That is a complex intuitionistic fuzzy function between two complex intu-

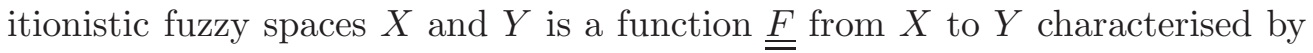
the ordered triple:

$$
\left(F(x),\left\{\underline{f}_{x}\right\}_{x \in X},\left\{\bar{f}_{x}\right\}_{x \in X}\right),
$$

where $F(x)$ is a function from $X$ to $Y$ and $\left\{\underline{f}_{x}\right\}_{x \in X},\{\bar{f} x\}_{x \in X}$ are family of functions from $E^{2}$ to $E^{2}$ satisfying the conditions (i) and (ii) such that the image of any complex intuitionistic fuzzy subset $A$ of the complex intuitionistic fuzzy space $X$ under $\underline{\underline{F}}$ is the intuitionistic fuzzy subset $F(A)$ of the intuitionistic fuzzy space $Y$ defined by:

$$
\underline{\underline{F}}(A) y= \begin{cases}\left(\vee_{x \in F^{-1}(y)} \underline{f}_{x}\left(r e^{i \theta_{r}}\right), \wedge_{x \in F^{-1}(y)} \bar{f}_{x}\left(\bar{r} e^{i \bar{\theta}_{r}}\right)\right), & \text { if } F^{-1}(y) \neq \phi \\ (0,1), & \text { if } F^{-1}(y)=\phi .\end{cases}
$$

We will call the functions $\underline{f}_{x}, \bar{f}_{x}$ the co-membership functions and the cononmembership functions, respectively. The complex intuitionistic fuzzy function $\underline{\underline{F}}$ will be denoted by $\underline{\underline{F}}=\left(F, f_{x}, \bar{f}_{x}\right)$.

Definition 2.10. (see R. Husban and Salleh 2016) A complex intuitionistic fuzzy binary operation $\underline{\underline{F}}$ on a complex intuitionistic fuzzy space $\left(X, E^{2}, E^{2}\right)$ 
is a complex intuitionistic fuzzy function $\underline{\underline{F}}:\left(X, E^{2}, E^{2}\right) \times\left(X, E^{2}, E^{2}\right) \rightarrow$ $\left(X, E^{2}, E^{2}\right)$ with co-membership functions $\underline{\underline{f}}_{x y}$ and co-nonmembership functions $\bar{f}_{x y}$ satisfying:

1. $\bar{f}_{x y}\left(r e^{i \theta_{r}}, s e^{i \theta_{s}}\right) \neq 0$ if and only if $r \neq 0, \theta_{r} \neq 0$ and $s \neq 0, \theta_{s} \neq 0$ and $\bar{f}_{x y}\left(w e^{i \theta_{w}}, z e^{i \theta_{z}}\right) \neq 1$ if and only if $w \neq 1, \theta_{w} \neq 2 \pi$ and $z \neq 1, \theta_{z} \neq 2 \pi$.

2. $\underline{f}_{x y}\left(E^{2} \times E^{2}\right)=E^{2}$ and $\bar{f}_{x y}\left(E^{2} \times E^{2}\right)=E^{2}, \underline{f}_{x y}, \bar{f}_{x y}$ are onto.

Definition 2.11. (see R .Husban and Salleh 2016) A complex intuitionistic fuzzy group is a complex intuitionistic fuzzy monoid in which each complex intuitionistic fuzzy element has an inverse. That is, a complex intuitionistic fuzzy groupoid $\left(\left(G, E^{2}, E^{2}\right) ; \underline{\underline{F}}\right)$ is a complex intuitionistic fuzzy group if and only if the following conditions are satisfied:

1. Associativity: for any choice of $\left(x, E^{2}, E^{2}\right),\left(y, E^{2}, E^{2}\right),\left(z, E^{2}, E^{2}\right)$ in

$$
\begin{aligned}
\left.\left(G, E^{2}, E^{2}\right) ; \underline{\underline{F}}\right)\left(\left(x, E^{2}, E^{2}\right) \underline{\underline{F}}\right. & \left.\left(y, E^{2}, E^{2}\right)\right) \underline{\underline{F}}\left(z, E^{2}, E^{2}\right) \\
& =\left(x, E^{2}, E^{2}\right) \underline{\underline{F}}\left(\left(y, E^{2}, E^{2}\right) \underline{\underline{F}}\left(z, E^{2}, E^{2}\right)\right) .
\end{aligned}
$$

2. Existence of an identity: There exists a complex intuitionistic fuzzy element $\left(e, E^{2}, E^{2}\right) \in\left(G, E^{2}, E^{2}\right)$ such that for all $\left(X, E^{2}, E^{2}\right)$ in $\left(\left(G, E^{2}, E^{2}\right)\right.$, $\underline{\underline{F}})$ :

$$
\left(e, E^{2}, E^{2}\right) \underline{\underline{F}}\left(x, E^{2}, E^{2}\right)=\left(x, E^{2}, E^{2}\right) \underline{\underline{F}}\left(e, E^{2}, E^{2}\right)=\left(x, E^{2}, E^{2}\right) .
$$

3. Existence of an inverse: for every complex intuitionistic fuzzy element $\left(x, E^{2}, E^{2}\right)$ in $\left(\left(G, E^{2}, E^{2}\right) ; \underline{\underline{F}}\right)$ : there exists a complex intuitionistic fuzzy element $\left(x^{-1}, E^{2}, E^{2}\right)$ in $\left(\left(\overline{\bar{G}, E^{2}}, E^{2}\right), \underline{\underline{F}}\right)$ such that:

$$
\left(x, E^{2}, E^{2}\right) \underline{\underline{F}}\left(x^{-1}, E^{2}, E^{2}\right)=\left(x^{-1}, E^{2}, E^{2}\right) \underline{\underline{F}}\left(x, E^{2}, E^{2}\right)=\left(e, E^{2}, E^{2}\right) .
$$

A complex intuitionistic fuzzy group $\left(\left(G, E^{2}, E^{2}\right), \underline{\underline{F}}\right)$ is called an abelian (commutative) complex intuitionistic fuzzy group if and only if for all $\left(x, E^{2}, E^{2}\right)$, $\left(y, E^{2}, E^{2}\right) \in\left(\left(G, E^{2}, E^{2}\right) ; \underline{\underline{F}}\right)$

$$
\left(x, E^{2}, E^{2}\right) \underline{\underline{F}}\left(y, E^{2}, E^{2}\right)=\left(y, E^{2}, E^{2}\right) \underline{\underline{F}}\left(x, E^{2}, E^{2}\right) .
$$

Definition 2.12. (see R. Husban, Salleh 2016) Let $S$ be a complex intuitionistic fuzzy subspace of the complex intuitionistic fuzzy space $\left(G, E^{2}, E^{2}\right)$. 
The ordered pair $(S ; \underline{\underline{F}})$ is a complex intuitionistic fuzzy subgroup of the complex intuitionistic fuzzy group $(S ; \underline{\underline{F}}) \leq\left(\left(G, E^{2}, E^{2}\right) ; \underline{\underline{F}}\right)$, denoted by $(S ; \underline{\underline{F}}) \leq$ $\left(\left(G, E^{2}, E^{2}\right) ; \underline{\underline{F}}\right)$, if $(S ; \underline{\underline{F}})$ defines a complex intuitionistic fuzzy group under the complex intuitionistic fuzzy binary operation $\underline{\underline{F}}$.

Obviously, if $(S ; \underline{\underline{F}})$ is a complex intuitionistic fuzzy subgroup of $\left(\left(G, E^{2}, E^{2}\right)\right.$; $\underline{\underline{F}})$ and $(T ; \underline{\underline{F}})$ is a complex intuitionistic fuzzy subgroup of $(S, \underline{\underline{F}})$, then $(\overline{\bar{T}} ; F)$ is a complex intuitionistic fuzzy subgroup of $\left(\left(G, E^{2}, E^{2}\right) ; \underline{\underline{F}}\right)$. Also if $\left(\left(G, E^{2}, E^{2}\right) ; \underline{\underline{F}}\right)$ is a complex intuitionistic fuzzy group with a complex intuitionistic fuzzy identity then both $\left\{\left(e, E^{2}, E^{2}\right)\right\}$ and $\left(\left(G, E^{2}, E^{2}\right) ; \underline{\underline{F}}\right)$ are complex intuitionistic fuzzy subgroups of complex intuitionistic fuzzy groups $\left(\left(G, E^{2}, E^{2}\right)\right.$; $\underline{\underline{F}})$.

\section{Complex Intuitionistic Fuzzy Normal Subgroup}

In this section we will introduce the notion of the associated complex intuitionistic fuzzy subgroups and a complex intuitionistic fuzzy normal subgroup based on associated complex intuitionistic fuzzy subgroup to obtain an interesting result regarding abelian complex intuitionistic fuzzy groups and related complex intuitionistic fuzzy normal subgroup.

Let $\left(\left(X, E^{2}, E^{2}\right) ; \underline{\underline{F}}\right)$ be a complex intuitionistic fuzzy group and

$$
U=\left\{\left(x, r_{x} e^{i \theta_{x}}, s_{x} e^{i \theta_{x}}\right): x \in U_{0}\right\}
$$

be a complex fuzzy intuitionistic subgroup of $\left(\left(X, E^{2}, E^{2}\right) ; \underline{\underline{F}}\right)$. Contrary to the ordinary case, the complex intuitionistic fuzzy elements $\left(x, r_{x} e^{i \theta_{x}}, s_{x} e^{i \theta_{x}}\right)$ of the complex intuitionistic fuzzy subgroup $(U, \underline{\underline{F}})$ are not necessary associative with the complex element $\left(X, E^{2}, E^{2}\right)$ of the complex intuitionistic fuzzy group $\left(\left(X, E^{2}, E^{2}\right) ; \underline{\underline{F}}\right)$ that is

$$
\begin{aligned}
\left(\left(x, E^{2}, E^{2}\right) \underline{\underline{F}}\left(y, E^{2}, E^{2}\right)\right) \underline{\underline{F}}\left(z, E^{2},\right. & \left.E^{2}\right) \\
& \neq\left(x, E^{2}, E^{2}\right) \underline{\underline{F}}\left(\left(y, E^{2}, E^{2}\right) \underline{\underline{F}}\left(z, E^{2}, E^{2}\right)\right),
\end{aligned}
$$

where $\left(x, E^{2}, E^{2}\right),\left(y, E^{2}, E^{2}\right)$ and $\left(z, E^{2}, E^{2}\right)$ are some complex intuitionistic fuzzy elements $U$ or $\left(X, E^{2}, E^{2}\right)$ such that one or two of this complex fuzzy intuitionistic element belong to $U$.

The following is an example of is not define associative complex intuitionistic fuzzy subgroup of complex intuitionistic fuzzy group.

Example 3.1. Let $X=\{1,-1, i,-i\}$ and $E^{2}=$ unit disc. Define the complex intuitionistic fuzzy binary operation $\underline{\underline{F}}=\left(F, \underline{f}_{x y}, \bar{f}_{x y}\right)$ on $\left(X, E^{2}, E^{2}\right)$, 
where $F: X \times X \rightarrow X$ is the ordinary multiplication of complex numbers. The co-memership and co-nonmemership functions have the following forms:

$$
\begin{aligned}
& \underline{f}_{11}\left(r e^{i \theta_{r}}, s e^{i \theta_{s}}\right)= \begin{cases}{\left[\frac{r s}{\alpha}\right] e^{i\left(\theta_{r} \wedge \theta_{s}\right)},} & \text { if } r s \leq \alpha^{2}, \\
{\left[1+\frac{r s-1}{1+\alpha}\right] e^{i\left(\theta_{r} \wedge \theta_{s}\right)},} & \text { if } r s>\alpha^{2},\end{cases} \\
& \bar{f}_{-11}\left(r e^{i \theta_{r}}, s e^{i \theta_{s}}\right)= \begin{cases}{\left[1-\frac{r s}{\alpha}\right] e^{i\left(\theta_{r} \wedge \theta_{s}\right)},} & \text { if } r s \leq \alpha^{2}, \\
{\left[\frac{1-r s}{1+\alpha}\right] e^{i\left(\theta_{r} \wedge \theta_{s}\right)},} & \text { if } r s>\alpha^{2},\end{cases} \\
& \underline{f}_{-11}\left(r e^{i \theta_{r}}, s e^{i \theta_{s}}\right)=\underline{f}_{1-1}\left(r e^{i \theta_{r}}, s e^{i \theta_{s}}\right) \\
& = \begin{cases}{\left[\frac{r s}{\alpha}\right] e^{i\left(\theta_{r} \wedge \theta_{s}\right)},} & \text { if } r s \leq \alpha \beta, \\
{\left[1+\frac{1-\beta}{1-\alpha \beta}(r s-1)\right] e^{i\left(\theta_{r} \wedge \theta_{s}\right)},} & \text { if } r s>\alpha \beta,\end{cases} \\
& \bar{f}_{-11}\left(r e^{i \theta_{r}}, s e^{i \theta_{s}}\right)=\bar{f}_{1-1}\left(r e^{i \theta_{r}}, s e^{i \theta_{s}}\right) \\
& = \begin{cases}{\left[1-\frac{r s}{\alpha}\right] e^{i\left(\theta_{r} \wedge \theta_{s}\right)},} & \text { if } r s \leq \alpha \beta, \\
{\left[\frac{1-\beta}{1-\alpha \beta}(1-r s)\right] e^{i\left(\theta_{r} \wedge \theta_{s}\right)},} & \text { if } r s \leq \alpha \beta,\end{cases}
\end{aligned}
$$

and the other co-membership and cononmemership functions are defined by the $r . s e^{i\left(\theta_{r} \wedge \theta_{s}\right)}$ where $\alpha$ and $\beta$ are given fixed real number such that $0<\beta<$ $\alpha<1$ and $\theta_{r}, \theta_{s} \in[0,2 \pi]$. Clearly $\left(\left(X, E^{2}, E^{2}\right) ; \underline{\underline{F}}\right)$ defines a non-uniform complex intuitionistic fuzzy group. Also the complex intuitionistic fuzzy subspace $U=\left\{\left(-1, \beta e^{i \theta_{\beta}}, 1 e^{i 2 \pi}\right),\left(1, \alpha e^{i \theta_{\alpha}}, 1 e^{i 2 \pi}\right)\right\}$ together with the complex intuitionistic fuzzy binary operation of $\underline{\underline{F}}$ define complex intuitionistic fuzzy subgroup $\left(\left(X, E^{2}, E^{2}\right) ; \underline{\underline{F}}\right)$. Now

$$
\begin{aligned}
\left(\left(1,1 e^{2 \pi i}, 1 e^{2 \pi i}\right)\right. & \underline{\underline{F}}\left(\left(1, \alpha e^{1.3 \pi i}, 1 e^{2 \pi i}\right) \underline{\underline{F}}\left(-1, \beta e^{\frac{\pi}{2} i}, 1 e^{2 \pi i}\right)\right. \\
= & \left(-1,\left(1-\frac{1-\beta}{1-\alpha \beta}\left(1-\frac{2 \alpha \beta}{1+\alpha}\right)\right) e^{\frac{\pi}{2} i},\left(\frac{1-\beta}{1-\alpha \beta}\left(1-\frac{\beta-\alpha \beta}{1+\alpha}\right)\right) e^{\frac{\pi}{2} i}\right),
\end{aligned}
$$

On the orher hand 


$$
\begin{aligned}
\left(1,1 e^{2 \pi i}, 1 e^{2 \pi i}\right) \underline{\underline{F}}\left(\left(1, \alpha e^{1.3 \pi i}, 1 e^{i 2 \pi i}\right) \underline{\underline{F}}\left(-1, \beta e^{\frac{\pi}{2} i}, 1 e^{i 2 \pi i}\right)\right) \\
=\left(-1,\left(0,1-\frac{(1-\beta)^{2}}{1-\alpha \beta}\right) e^{\frac{\pi}{2} i},\left(1-\frac{(1-\beta)^{2}}{1-\alpha \beta}\right) e^{\frac{\pi}{2} i}\right) .
\end{aligned}
$$

That is, the complex intuitionistic fuzzy elements of $U$ are not associative with complex intuitionistic fuzzy elements of complex intuitionistic fuzzy spaces $\left(X, E^{2}, E^{2}\right)$.

Definition 3. An associative complex intuitionistic fuzzy subgroup $(U, \underline{\underline{F}})$ of the complex intuitionistic fuzzy group $\left(\left(X, E^{2}, E^{2}\right) ; \underline{\underline{F}}\right)$ is a complex intuitionistic fuzzy group $(U, \underline{\underline{F}})$ of $\left(\left(X, E^{2}, E^{2}\right) ; \underline{\underline{F}}\right)$ in which complex intuitionistic fuzzy elements of $U$ are associative with complex intuitionistic fuzzy elements of $\left(X, E^{2}, E^{2}\right)$ for any arbitrary choice of complex intuitionistic fuzzy elements of $U$ and $\left(X, E^{2}, E^{2}\right)$.

Example 3.2. Let $X=\{-1,1,-i, i\}$. Defined the complex intuitionistic fuzzy binary operation $\underline{\underline{F}}=\left(F, f_{x y}\right)$ on $\left(X, E^{2}, E^{2}\right)$ such that $F: X \times X \rightarrow$ $X$ is the ordinary multiplication of complex numbers and the co-membership function are given for all $x, y \in X$ by

$$
\underline{f}_{x y}\left(\underline{r} e^{i \underline{\theta}_{r}}, s e^{i \theta_{s}}\right)=(r \wedge s) e^{i\left(\theta_{r} \wedge \theta_{s}\right)}, \bar{f}_{x y}\left(r e^{i \bar{\theta}_{r}}, s e^{i \bar{\theta}_{s}}\right)=(r \wedge s) e^{i\left(\bar{\theta}_{r} \wedge \bar{\theta}_{s}\right)} .
$$

Obviously the complex intuitionistic fuzzy spaces

$$
U=\left\{\left(1,0.5 e^{2 \pi i}, 0.4 e^{1.5 \pi i}\right),\left(-1,0.5 e^{\pi i}, 0.4 e^{\pi i}\right)\right\}
$$

defines an associative complex intuitionistic fuzzy subgroup of complex intuitionistic fuzzy group $\left(\left(X, E^{2}, E^{2}\right) ; \underline{\underline{F}}\right)$ under $\underline{\underline{F}}$.

Theorem 3.1. Let $\left(\left(X, E^{2}, E^{2}\right) ; \underline{\underline{F}}\right)$ with $\underline{\underline{F}}=(F, \underline{f}, \bar{f})$ be uniform complex intuitionistic fuzzy group if $\underline{f}\left(1 e^{\overline{\overline{i \theta}}}, r e^{i \theta_{r}}\right)=\underline{f}\left(r e^{\overline{i \theta_{r}}}, 1 e^{i \theta}\right)=r e^{i \theta_{r}}$, and $\bar{f}\left(1 e^{i \bar{\theta}}, r e^{i \bar{\theta}_{r}}\right)=\bar{f}\left(r e^{i \bar{\theta}_{r}}, 1 e^{i \bar{\theta}}\right)=r e^{i \bar{\theta}_{r}}$ then every complex intuitionistic fuzzy subgroup of the complex intuitionistic fuzzy group $\left(\left(X, E^{2}, E^{2}\right) ; \underline{\underline{F}}\right)$ is an associative complex intuitionistic fuzzy subgroup.

Proof. Let $(U, \underline{\underline{F}})$ be a complex intuitionistic fuzzy subgroup of complex intuitionistic fuzzy group of $\left(\left(X, E^{2}, E^{2}\right) ; \underline{F}\right)$ Consider the complex intuitionistic fuzzy elements $\left(x, E^{2}, E^{2}\right),\left(y, E^{2}, E^{2}\right)$ and $\left(z, E^{2}, E^{2}\right)$ of $U$ or $\left(X, E^{2}, E^{2}\right)$ such that one ore two of these complex intuitionistic fuzzy elements belong to $U$ Now using the properties of $\underline{f}, \bar{f}$ and the associativity of $\underline{\underline{F}}$ we have:

$$
\left(x, E^{2}, E^{2}\right) \underline{\underline{F}}\left(\left(y, E^{2}, E^{2}\right) \underline{\underline{F}}\left(z, E^{2}, E^{2}\right)\right)=\left(x, E^{2}, E^{2}\right) \underline{\underline{F}}
$$




$$
\begin{aligned}
& \left(\left(y F z, \underline{f}\left(E^{2} \times E^{2}\right), \bar{f}\left(E^{2} \times E^{2}\right)\right)\right. \\
= & \left(x, E^{2}, E^{2}\right) \underline{\underline{F}}\left(\left(y F z, E^{2}, E^{2}\right)\right) \\
= & \left(x F(y F z), \underline{f}\left(E^{2} \times E^{2}\right), \bar{f}\left(E^{2} \times E^{2}\right)\right) \\
= & \left((x F y) F z, E^{2}, E^{2}\right) \\
= & \left(\left(x, E^{2}\right) \underline{\underline{F}}\left(y, E^{2}, E^{2}\right)\right) \underline{\underline{F}}\left(z, E^{2}, E^{2}\right) .
\end{aligned}
$$

which proves the associativity of intuitionistic fuzzy members of $U$ with complex intuitionistic fuzzy elements of the complex intuitionistic fuzzy space $\left(X, E^{2}, E^{2}\right)$ under $\underline{\underline{F}}$.

Definition 3.2. The complex intuitionistic fuzzy subgroup $U$ of the complex intuitionistic fuzzy group $\left(\left(X, E^{2}, E^{2}\right) ; \underline{\underline{F}}\right)$ is called a complex intuitionistic fuzzy normal subgroup if:

(i) $U$ is associative in $\left(\left(X, E^{2}, E^{2}\right) ; \underline{\underline{F}}\right)$.

(ii) $\left(x, E^{2}, E^{2}\right) U=U\left(x, E^{2}, E^{2}\right)$, such that $x \in X$.

Theorem 3.2. If $U=\left\{\left(z, r_{z} e^{i \theta_{r_{z}}}, \bar{r}_{z} e^{i \bar{\theta}_{r_{z}}}\right): z \in U_{0}\right\}$ is a complex intuitionistic fuzzy subgroup of the complex intuitionistic fuzzy group $\left(\left(X, E^{2}, E^{2}\right), \underline{\underline{F}}\right)$ then Uis a complex intuitionistic fuzzy normal subgroup if and only if:

1. $\left(U_{0}, F\right)$ is a intuitionistic fuzzy normal subgroup of the intuitionistic fuzzy group $((X, I, I), \mathbf{F})$,

(ii) $\underline{f}_{x z}\left(E^{2}, r_{z} e^{i \theta_{z}}\right)=\underline{f}_{z^{\prime} x}\left(r_{z^{\prime}} e^{i \theta_{z^{\prime}}}, E^{2}\right), \bar{f}_{x z}\left(E^{2}, \bar{r}_{z} e^{i \bar{\theta}_{z}}\right)=\bar{f}_{z^{\prime} x}\left(\bar{r}_{z^{\prime}} e^{i \bar{\theta}_{z^{\prime}}}, E^{2}\right): x F z=$ $z^{\prime} F x$ and $z, z^{\prime} \in U_{0}$. where $x \in X$.

Proof. Assume $U=\left\{\left(z, r_{z} e^{i \theta_{r z}}, \bar{r}_{z} e^{i \bar{\theta}_{r z}}\right): z \in U_{0}\right\}$ is a complex intuitionistic fuzzy normal subgroup of the complex intuitionistic fuzzy group $\left(\left(X, E^{2}, E^{2}\right), \underline{\underline{F}}\right)$ from the correspondence theorem we have $\left(U_{0}, \mathbf{F}\right)$ is an intuitionistic normal subgroup of the intuitionistic fuzzy group $((X, I, I), \mathbf{F})$, Using the normality of $U$ we have $\left(x, E^{2}, E^{2}\right) U=U\left(x, E^{2}, E^{2}\right): x \in X$. That is

$$
\begin{aligned}
\left\{\left(x F z, f_{x z}\left(E^{2}, r_{z} e^{i \theta_{r z}}\right)\right.\right. & \left.\left., \bar{f}_{x z}\left(E^{2}, \bar{r}_{z} e^{i \bar{\theta}_{r z}}\right)\right): z \in U_{0}\right\} \\
& =\left\{\left(z F x, f_{z x}\left(r_{z} e^{i \theta_{r z}}, E^{2}\right), \bar{f}_{z x}\left(\bar{r}_{z} e^{i \bar{\theta}_{r z}}, E^{2}\right)\right): z \in U_{0}\right\} .
\end{aligned}
$$

Therefore, for every $z \in U_{0}$ there exists $z^{\prime} \in U_{0}$ such that $x F z=z^{\prime} F x$. In other words $x F U_{0}=U_{0} F x$. Hence $U_{0}$ is an ordinary normal subgroup of ordinary group $(X, F)$

Hence, we prove (i). (ii) follows directly from the definition. The other part of the proof is direct. 


\section{Conclusion}

In this paper, we generalized the study initiated in Fathi and salleh (2011) about intuitionistic fuzzy normal subgroup to the context of complex intuitionistic fuzzy normal subgroup. We define the notion of a complex intuitionistic fuzzy normal subgroup using the notion of a complex intuitionistic fuzzy space.

\section{References}

[1] Abdul Razak Salleh, Sifat-sifat homomorfisma kabur bagi kumpulan kabur, In: Prosiding Simposium Kebangsaan Sains Matematik ke-7, Shah Alam, Institut Teknologi Mara, 1996.

[2] Abd Ulazeez Alkouri, Abdul Razak Salleh, Complex intuitionistic fuzzy set, In: AIP Conf. Proceedings. 12-14 June, Kuala Lumpur, Malaysia, 1482 (2012), 464-470, doi: 10.1063/1.4757515.

[3] A. Al-Husban, A.R. Salleh, Complex fuzzy group based on complex fuzzy space, Global Journal of Pure and Applied Mathematics, 2, No. 12 (2016), 1433-1450.

[4] A. Al-Husban, A.R. Salleh, N. Hassan, Complex fuzzy normal subgroup, In: The UKM FST Postgraduate Colloquium, AIP Conference Proceedings (2015), 060008.

[5] R. Al-Husban, A.R. Salleh, A.G. Ahmad, Complex intuitionistic fuzzy rings, In: AIP Conference Proceedings, 1739, No. 1 (2016).

[6] R. Al-Husban, A.R. Salleh, A.G. Ahmad, Complex intuitionistic fuzzy subrings, In: AIP Conference Proceedings, 1784, No. 1 (2016).

[7] J.M. Anthony, H. Sherwood, Fuzzy groups redefined, Journal of Math. Anal. Appl., 69 (1979), 124-130.

[8] J.M. Anthony, H. Sherwood, A characterization of fuzzy subgroups, Fuzzy Sets and Systems, 7 (1982), 297-305.

[9] J.J. Buckley, Fuzzy complex numbers, Fuzzy Sets and Systems,33 (1989), 333-345.

[10] K.A. Dib, A.A.M. Hassan, The fuzzy normal subgroup, Fuzzy Sets and Systems, 98 (1998), 393-402.

[11] K.A. Dib, On fuzzy spaces and fuzzy group theory, Inform. Sci., 80 (1994), 253-282.

[12] M. Fathi, A.R. Salleh, Intuitionistic fuzzy groups, Asian Journal of Algebra, 2, No. 1 (2009), 1-10.

[13] M. Fathi, A.R. Salleh, The intuitionistic fuzzy normal subgroup, International Journal of Fuzzy Logic and Intelligent Systems, 10, No. 1 (2010), 82-88.

[14] I.J. Kumar, P.K. Saxena, P. Yadava, Fuzzy normal subgroups and fuzzy quotients, Fuzzy Sets and Systems, 46 (1992), 121-132.

[15] C.V. Negoita, D.A. Ralescu, Applications of Fuzzy Sets to System Analysis, Wiley, New York, 1975.

[16] D. Ramot, M. Friedman, G. Langholz, A. Kandel, Complex fuzzy logic, IEEE Transaction on Fuzzy Systems, 11, No. 4 (2003), 450-461. 
[17] D. Ramot, R. Milo, M. Friedman, A. Kandel, Complex fuzzy sets, IEEE Transaction on Fuzzy Systems, 10 (2002), 171-186.

[18] A. Ronsfeld, Fuzzy groups, Journal of Mathematical Analysis and Applications, 35 (1971), 512-517.

[19] H. Sherwood, Product of fuzzy subgroups, Fuzzy Sets and Systems, 11 (1983), 79-89.

[20] L.A. Zadeh, Fuzzy sets, Inform. Control, 8 (1965), 338-353.

[21] P. Zhan, Z. Tan, Intuitionistic M-fuzzy subgroups, Soochow Journal of Mathematics, 30 (2004), 85-90. 